\title{
THE ELECTROPRODUCTION OF ETAS AND KAONS
}

\author{
O.K. BAKER \\ Department of Physics, Hampton University, Hampton, VA 23668, \\ and Physics Division, Jefferson Lab, Newport News, VA 23606 \\ E-mail: baker@jlab.org
}

\begin{abstract}
Experimental results for the electromagnetic production of eta and $K$ mesons are compared with QCD-inspired models. The eta mesons from the decay of the $S_{11}$ resonance were used to study the momentum transfer dependence of the relevant helicity amplitude and cross section in the reaction ${ }^{1} \mathrm{H}\left(e, e^{\prime} \mathrm{p}\right) \eta$. The ${ }^{1} \mathrm{H}\left(\mathrm{e}, \mathrm{e}^{\prime} \mathrm{K}^{+}\right) \Lambda$ reaction was studied as a function of squared four-momentum transfer, $Q^{2}$, and of the virtual photon polarization parameter, $\epsilon$. Both of these experiments were performed at Jefferson Lab during the early years of operation. The new precision data serve to constrain model calculations and provide new insights into the physical processes.
\end{abstract}

\section{General Considerations}

To the extent that quark masses can be ignored, the QCD Lagrangian may be separated into terms involving only left- handed quarks and terms involving only right-handed quarks. If attention is limited to the three lightest flavors, this implies an ungauged symmetry of the Lagrangian density under the direct product of global SU(3) flavor rotations on the left- and right-handed fields. This invariance under $\mathrm{SU}(3)_{\mathrm{L}} \mathrm{xSU}(3)_{\mathrm{R}}$ is an important (approximate) symmetry of the strong interaction ${ }^{3}$. With three massless quarks, $(u, d, s)$, the QCD Lagrangian is invariant under this global, chiral $\mathrm{SU}(3)_{\mathrm{L}} \times \mathrm{SU}(3)_{\mathrm{R}}$ symmetry. Pseudoscalar mesons are thought to be approximate Goldstone bosons resulting from the spontaneous breaking of this global symmetry ${ }^{2}$.

Two of these pseudoscalar mesons, the kaon and the eta mesons, are considered in this manuscript. These mesons (along with several others) are the subject of intense experimental and theoretical study at Jefferson Lab. The insights resulting from the work in Hall $\mathrm{C}$ will be described here.

The kinematic quantities relavent to the discussion of pseudoscalar production are defined using standard conventions in the literature. The reactions studied are summarized as

$$
e+p \rightarrow e^{\prime}+m+B
$$

where $\mathrm{e}$ and $\mathrm{e}^{\prime}$ are the incident and scattered electron, $\mathrm{p}$ represents the proton target (a liquid hydrogen target was used in all of these measurements), $\mathrm{m}$ is 
the electroproduced pseudoscalar meson, and B is the associated hyperon (in the case of kaon electroproduction) or baryon (in the case of eta electroproduction) produced in the reaction. The incident $\left(\mathrm{e}=\left(\mathrm{E}, \vec{p}_{e}\right)\right)$ and scattered electrons $\left(\mathrm{e}^{\prime}=\left(\mathrm{E}_{\mathrm{e}^{\prime}}, \overrightarrow{\mathrm{p}}_{\mathbf{e}^{\prime}}\right)\right)$ define the electron scattering plane. The recoiling meson $\mathrm{m}=\left(\mathrm{E}_{\mathrm{m}}, \overrightarrow{\mathrm{p}}_{\mathrm{m}}\right)$ and residual system, $\mathrm{B}=\left(\mathrm{E}_{\mathrm{B}}, \overrightarrow{\mathrm{P}}_{\mathrm{B}}\right)\left(\mathrm{a} \Lambda\right.$ or $\Sigma^{0}$ hyperon in the case of kaon electroproduction and a proton in the case of eta production) define the production plane. The virtual photon that is emitted lies in both of these planes. The angle between the electron scattering and production planes, the out-of-plane angle, is denoted by $\phi$. The polar angle between the virtual photon and the pseudoscalar meson is denoted by $\theta_{m \gamma}$ while the angle between the incident and scattered electron is $\theta_{\mathrm{e}}$. The four momentum transfer from the electron to the proton is denoted by $q=e-e^{\prime}$. Its components are $E-E_{\mathrm{e}^{\prime}}=\nu$ (the electron energy loss in the laboratory system) and $\overrightarrow{\mathrm{q}}=\overrightarrow{\mathrm{p}}_{\mathrm{e}}-\overrightarrow{\mathrm{p}}_{\mathrm{e}^{\prime}}$. The square of the four momentum transfer $\mathrm{q}^{2}=-\mathrm{Q}^{2}=-4 \mathrm{EE}_{\mathrm{e}} \sin ^{2}\left(\theta_{\mathrm{e}} / 2\right)$. Other quantities of interest are $\mathrm{W}^{2}=\mathrm{s}=\mathrm{M}_{\mathrm{p}}^{2}+2 \mathrm{M}_{\mathrm{p}} \nu-\mathrm{Q}^{2}$, the squared mass of the system recoiling against the electron (that is, the photon-proton system), $t=(q-m)^{2}=q^{2}+M_{m}^{2}-2 q m$, and $u=(m-p)^{2}$. Here $s, t$, and $u$ are the Mandelstam variables.

The expression for the cross section for electromagnetic production of pseudoscalar mesons involves terms that are functions of the angles, virtual photon polarization and Mandelstam variables. The expression for the cross section may be simplified in the case when the meson is detected at $\theta_{\mathrm{m} \gamma}=0$, and integration over all azimuthal angles $\phi$, that is for parallel kinematics. In this case, for an unpolarized target, no incident electron polarization, no final state polarization detected, and in the one-photon exchange approximation, the laboratory cross section may be related to that in the center of mass by the expression ${ }^{3}$

$$
\frac{d^{5} \sigma}{d E_{e^{\prime}} d \Omega_{e^{\prime}} d \Omega_{m}}=\Gamma\left(\frac{d \sigma_{T}}{d \Omega_{m}}+\epsilon \frac{d \sigma_{L}}{d \Omega_{m}}\right)
$$

where $\Gamma$ is the virtual photon flux and $\epsilon$ is the virtual photon polarization parameter ${ }^{3}$. The two terms on the right hand side of (2) contain the transverse and longitudinal cross sections. This expression represents the starting point for the comparison of data to model calculations in the subsequent discussion.

\section{Eta Production}

One of the more sensitive and fundamental quark model tests is the $\mathrm{Q}^{2}$ dependences of the coupling strengths. For the present discussion of eta production, 
these couplings are conveniently expressed in terms of the transition matrix elements between states of different helicity.

The $S_{11}(1535)$ provides a unique opportunity to study the production of the eta meson ${ }^{4}$. In the resonance region there are a number of hadronic resonances that are produced by the electromagnetic interaction. The overlap between these resonances makes a study of a single state difficult, particularly if it is not produced strongly compared to its nearest neighbor. The $S_{11}$ may be isolated experimentally because the decay to the proton-eta channel dominates over all other decay fractions. Spin and isospin selection rules prevent $\Delta$ resonances from decaying into $\mathrm{p}-\eta$. Additionally, the $\mathrm{S}_{11}$ is the only isospin- $\frac{1}{2}$ baryon resonance in the vicinity of $1535 \mathrm{MeV}$ to decay strongly in this way ${ }^{4}$.

In making a comparison of the individual resonance contributions to the cross sections, it is convenient to express the cross sections in terms of its multipole expansion. At the energies considered here, it is appropriate to retain only $\mathrm{S}, \mathrm{P}$, and $\mathrm{D}$-waves that are proportional to the dominant $\mathrm{E}_{\mathrm{0}+}$ and $\mathrm{S}_{0+}$ multipoles. The cross section for eta production may then be written as ${ }^{4}$

$$
\frac{d^{2} \sigma_{T}}{d \Omega_{\eta}^{*}}=\frac{\left|\vec{p}_{\eta}\right| W}{m_{p} K}\left\{\left|E_{0+}\right|^{2}-R e\left[E_{0+}^{*}\left\{2 \cos \theta_{\eta \gamma}^{*} M_{1}-\left(3 \cos ^{2} \theta_{\eta \gamma}^{*}-1\right)\left(E_{2-}-3 M_{2-}\right)\right\}\right]\right\}
$$

$$
\frac{d^{2} \sigma_{L}}{d \Omega_{\eta}^{*}}=\frac{Q^{2}\left|\vec{p}_{\eta}\right| W}{\left|\vec{q}^{*}\right|^{2} m_{p} K}\left\{\left|S_{0++}\right|^{2}+2 \operatorname{Re}\left[S_{0+}^{*}\left\{2 \cos \theta_{\eta \gamma}^{*} S_{1-}-2\left(1-3 \cos ^{2} \theta_{\eta \gamma}^{*}\right) S_{2-}\right\}\right]\right\}
$$

In this notation, $E_{l_{ \pm}}, M_{1 \pm}$, and $S_{I_{ \pm}}$denote electric, magnetic, and scaler excitation multipoles, respectively. The orbital angular momentum is given by $l$. The ' \pm ' gives the total angular momentum through the expression $j=l \pm \frac{1}{2}$. In the expressions (3) and (4) $\theta_{\eta \gamma}^{*}$ is the polar angle between the virtual photon and eta meson. The momentum of the eta in the center of mass of the hadronic resonance, $\left|\vec{p}_{\eta}^{*}\right|$, is given by

$$
\left|\overrightarrow{p_{\eta}}\right|=\frac{\left\{\left[W^{2}-\left(m_{p}+m_{\eta}\right)^{2}\right]\left[W^{2}-\left(m_{p}-m_{\eta}\right)^{2}\right]\right\}^{1 / 2}}{2 W}
$$

The particle helicity may be derived from these expressions and compared to calculations. The helicity $\lambda$ of a particle is defined to be the projection of its spin in its direction of motion. Massive particles with spin s can in general have $2 s+1$ helicity states $(-s,-s+1, \ldots, s-1, s)$ while massless objects are constrained by parity to have only the helicity values $\pm s$. So, for example, 


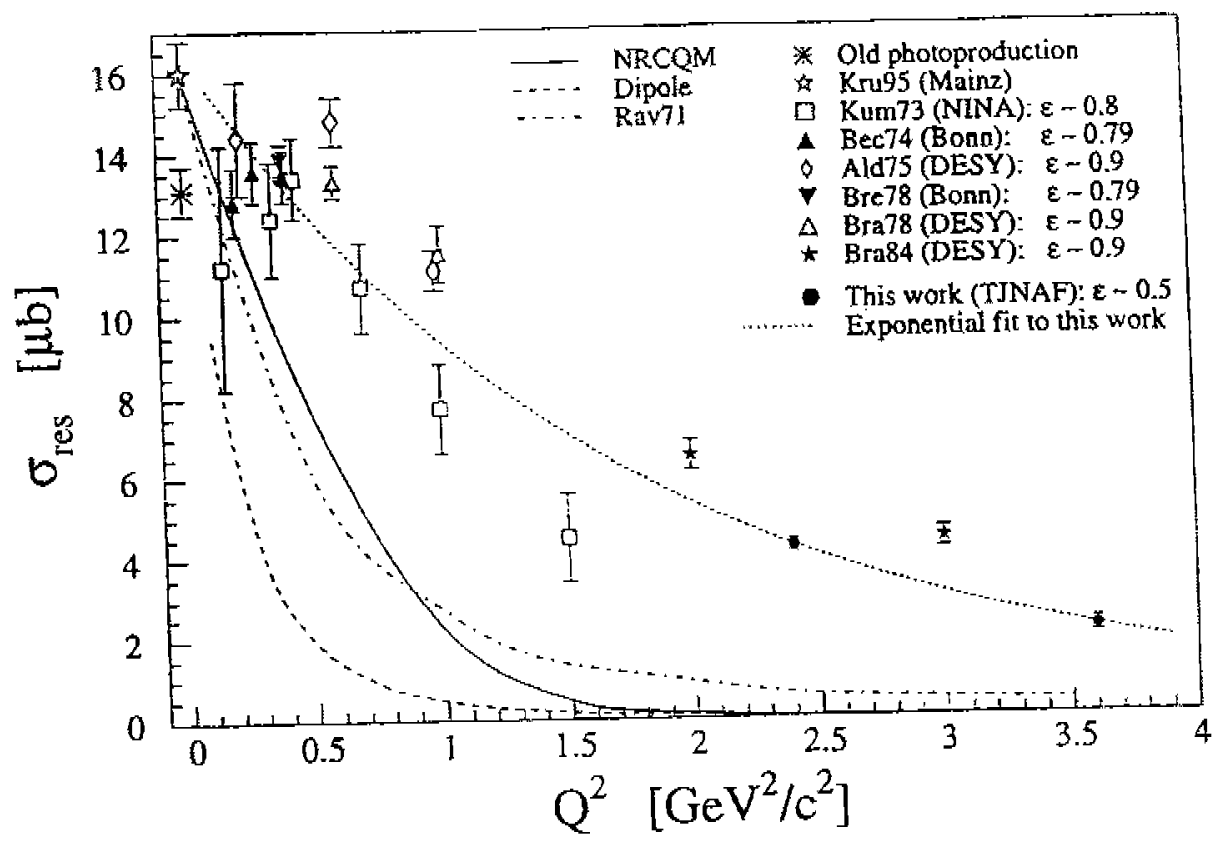

Figure 1. The world's data for the cross section $\sigma_{\mathrm{res}}\left(\mathrm{ep} \rightarrow \mathrm{e}^{\prime} \mathrm{p} \eta\right)$ at $\mathrm{W} \approx 1535 \mathrm{MeV}$ versus $Q^{2}$. The new Jefferson Lab data is shown as the full dots. The new data exhibits a slightly different $Q^{2}$ dependence than previous measurements in this kinematic range (high momentum transfers). The figure is taken from the dissertation of C. Armstrong.

massless quarks may have only the two helicity states in a world of unbroken chiral symmetry. For the present work, the stationary proton targets are states of helicity $\lambda= \pm \frac{1}{2}$.

In the case of a proton $\left(\mathrm{J}^{\mathrm{P}}=\frac{1}{2}^{+}\right)$excited to an $\mathrm{S}_{11}$ resonance $\left(\mathrm{J}^{\mathrm{P}}=\frac{1}{2}^{-}\right)$, the helicity amplitude of interest is $\mathrm{A}_{1 / 2}^{\mathrm{NN}^{*}}$. (In this notation, the helicity amplitude of an initial nucleon state with helicity $\lambda$ and final excited state nucleon resonance $\mathrm{N}^{*}$ with helicity $\lambda^{\prime}$ is denoted by $\mathrm{A}_{\lambda^{\prime}}^{\mathrm{NN}^{*}}$.) The amplitude $A_{1 / 2}$ for the process $\gamma p \rightarrow S_{11}(1535)$ is expressed in terms of the $S_{11}$ to the $\mathrm{E}_{0+}$ multipole at the resonance mass $\mathrm{W}=\mathrm{W}_{\mathrm{R}}$ by ${ }^{4}$ 


$$
\begin{aligned}
A_{1 / 2}= & {\left[(2 j+1) \pi \frac{\left|\vec{p}_{\eta}^{*}\right|_{R} W_{R}}{m_{p} K} \frac{W_{R}}{m_{p}} \frac{\Gamma_{R}^{2}}{\Gamma_{\eta}}\right]^{1 / 2} \operatorname{Im}\left[E_{0+}\left(W=W_{R}\right)\right] } \\
& =\left[2 \pi \frac{\left|\vec{p}_{\eta}^{*}\right|_{R} W_{R}}{m_{p} K} \frac{W_{R}}{m_{p}} \frac{\Gamma_{R}}{b_{\eta}}\right]^{1 / 2}\left|E_{0+}\left(W=W_{R}\right)\right|
\end{aligned}
$$

Here $\Gamma_{\eta}$ and $\Gamma_{R}$ are the partial and full widths of the resonance, and $b_{\eta}=\frac{\Gamma_{\eta}}{\Gamma_{\mathrm{R}}}$ is the eta branching fraction for the decay $S_{11}(1535) \rightarrow p \eta$, and $K$ is a kinematic factor.

If the contributions from resonances other than the $S_{11}$ are neglected, it can be shown that in parallel kinematics ${ }^{4}$

$$
\begin{gathered}
A_{1 / 2}=\left[\frac{W_{R} \Gamma_{R}}{2 m_{p} b_{\eta}} \sigma_{T}\left(W=W_{R}\right)\right]^{1 / 2} \\
=\left[\frac{W_{R} \Gamma_{R}}{2 m_{p} b_{\eta}} \frac{\sigma_{\text {res }}\left(W=W_{R}\right)}{1+\epsilon R}\right]^{1 / 2} .
\end{gathered}
$$

where $\mathrm{R}$ is the ratio of longitudinal to transverse cross section. The resonance was isolated experimentally by the reaction

$$
e+p \rightarrow e^{t}+S_{11}(1535)
$$

followed by the baryon decay

$$
S_{11} \rightarrow p+\eta
$$

The incident electron beam was directed onto a liquid hydrogen target. The scattered electron and the proton from the resonance decay were detected with the SOS and HMS respectively. The eta meson is identified by its missing mass.

The world's data for $\sigma_{\text {res }}\left(\mathrm{ep} \rightarrow \mathrm{e}^{\prime} \mathrm{p} \eta\right.$ ) (at the resonance mass) is shown in Fig. 1. The helicity amplitude $A_{1 / 2}$ is shown in Fig. 2. The data from the work in Hall $\mathrm{C}$ at Jefferson Lab is shown as the solid dots. The dominant uncertainty in the extraction of $A_{1 / 2}$ is the poor knowledge of the eta width and $\mathrm{p}-\eta$ branching fraction. The new data is somewhat lower in magnitude and has a slightly different trend than the other data at the higher $Q^{2}$.

All of the curves shown are CQM calculations. The references corresponding to the various calculations are $[\mathrm{Li90}]^{5},[\mathrm{War} 90]^{6},[\mathrm{Cap95}]^{7},[\mathrm{Sta95}]^{8}$. 


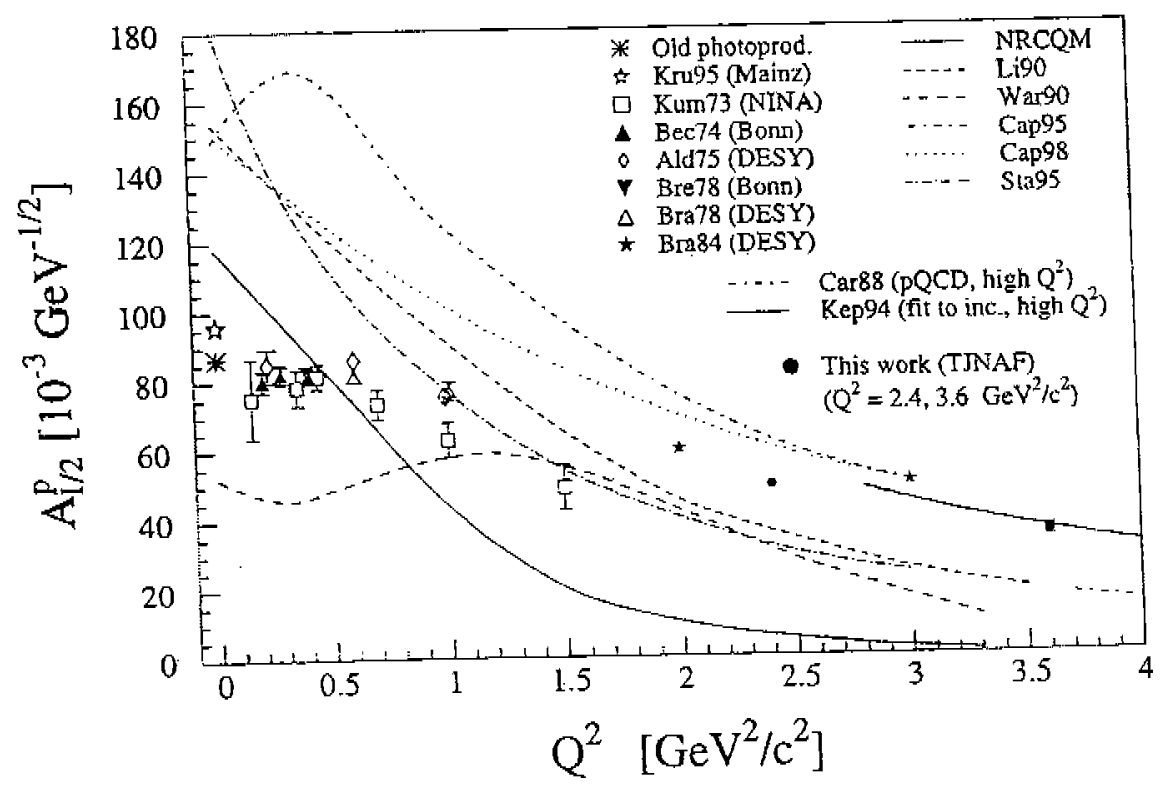

Figure 2. The world's data for $A_{1 / 2}^{P}\left(S_{11}(1535)\right)$, measured via the $p \eta$ channel, together with theoretical predictions labeled in the text. The new Jefferson Lab data is shown as the full dots. The new measurement exhibits a slightly different $Q^{2}$ dependence at high momentum transfers than previous results. The figure is taken from the dissertation of $C$. Armstrong

Only the curve labeled [War90] exhibits a stiff $\mathrm{Q}^{2}$ behavior resembling the trend of the data. One conclusion to draw from this comparison is that there is a need for refinement of the CQM as presented here in order to account for the experimentally observed $Q^{2}$ dependence of the $S_{11}(1535)$ resonance in the decay to proton and eta meson.

\section{Kaon Production}

The K-meson or kaon is the lightest quark-antiquark system in which a strange quark, $\mathrm{s}$, is paired with an up, $\mathrm{u}$, or down, d, quark. In recent years, a wealth of new theoretical studies of kaon electroproduction (and photoproduction as well) has emerged, fueled by the promise of understanding hadrons in 
terms of QCD and the construction of new accelerators capable of providing continuous-wave, high current, electron beams both with and without high polarization in the few GeV energy range $\begin{array}{llll}11 & 12 & 13 & 15\end{array}$

One of the first experimental studies performed at the new CEBAF accelerator at Jefferson Lab was the electroproduction of kaons. Experiment E91-016 and E93-018 at Jefferson Lab were designed to study the reactions

$$
\mathrm{e}+\mathrm{p} \rightarrow \mathrm{e}^{\prime}+\mathrm{K}^{+}+\Lambda \text { and } \mathrm{e}+\mathrm{p} \rightarrow \mathrm{e}^{\prime}+\mathrm{K}^{+}+\Sigma^{0} \text {. }
$$

Then by measuring the cross section at several values of $\epsilon$ while holding $\mathrm{Q}^{2}$, $W$, and $t$ constant, the longitudinal and transverse cross sections may be determined separately. Then the super-Rosenbluth separation was carried out to separate the longitudinal and transverse cross sections. Jefferson Lab Experiment E93-018 was the very first experiment to accurately determine these cross sections ${ }^{17}$.

The High Momentum Spectrometer (HMS) was used to detect the scattered electrons in a modest background of pions mainly, while the SOS was used to detect the electroproduced kaon in a high background of pions and protons. An unpolarized electron beam of between 2.4 and $4.0 \mathrm{GeV}$ was incident upon a liquid hydrogen target to produce the reactions. Shown in Table I are the kinematic settings for the data to be presented in this manuscript.

\begin{tabular}{|c|c|c|c|c|c|c|c|c|}
\hline$\overline{\mathrm{Q}}^{2}$ & $\mathrm{E}_{\mathrm{e}}$ & $\mathrm{E}_{\mathrm{e}^{\prime}}$ & $\mathrm{pK}_{\mathrm{K}}$ & $\theta_{\mathrm{e}}$ & $\theta_{\gamma}$ & $\mathrm{W}$ & $\epsilon$ & $-\mathrm{t}$ \\
\hline 0.52 & 2.445 & 0.833 & 1.126 & 29.27 & 13.33 & 1.84 & 0.55 & 0.22 \\
0.52 & 3.245 & 1.633 & 1.126 & 18.02 & 16.62 & 1.84 & 0.77 & 0.22 \\
0.52 & 4.045 & 2.433 & 1.126 & 13.20 & 18.34 & 1.84 & 0.86 & 0.22 \\
\hline 0.75 & 2.445 & 0.726 & 1.188 & 37.94 & 13.41 & 1.83 & 0.46 & 0.30 \\
0.75 & 3.245 & 1.526 & 1.188 & 22.44 & 17.62 & 1.83 & 0.72 & 0.30 \\
0.75 & 4.045 & 2.326 & 1.188 & 16.23 & 19.74 & 1.83 & 0.83 & 0.30 \\
\hline 1.00 & 2.445 & 0.635 & 1.216 & 47.30 & 13.05 & 1.81 & 0.38 & 0.41 \\
1.00 & 3.245 & 1.435 & 1.216 & 26.79 & 18.24 & 1.81 & 0.67 & 0.41 \\
1.00 & 4.045 & 2.235 & 1.216 & 19.14 & 20.77 & 1.81 & 0.81 & 0.41 \\
\hline 2.00 & 3.245 & 0.844 & 1.634 & 50.59 & 13.54 & 1.84 & 0.37 & 0.74 \\
2.00 & 3.545 & 1.144 & 1.634 & 41.11 & 15.66 & 1.84 & 0.48 & 0.74 \\
2.00 & 4.045 & 1.644 & 1.634 & 31.83 & 18.13 & 1.84 & 0.61 & 0.74 \\
\hline
\end{tabular}

\section{TABLE I}

Nominal Kinematics for Experiment E93018 energies in $\mathrm{GeV}$, momenta in $\mathrm{GeV} / \mathrm{c}$, angles in degrees 
Two peaks corresponding to the masses of the $\Lambda(1115)$ and the $\Sigma^{0}(1192)$ were clearly identified in the missing mass spectrum ${ }^{17}$. The production of higher mass hyperons were energetically forbidden in this measurement. In this study, only the reaction $\mathrm{e}+\mathrm{p} \rightarrow \mathrm{e}^{\prime}+\mathrm{K}^{+}+\Lambda$ is considered.

The systematic uncertainty in the measurement is typically $5 \%$ while the statistical uncertainty in all cases is about $2 \%$ or less. The systematic uncertainty is dominated by the uncertainties in spectrometer acceptances and kaon survival probability. The point-to-point random uncertainties which are more relevant for the longitudinal-transverse cross section separation are smaller since some of the larger contributors to the systematic uncertainty are strongly correlated between the various $\epsilon$ settings. In general, the pointto-point random uncertainties are at or below $3 \%$. Radiative corrections of up to $30 \%$ are applied to the final results ${ }^{17}$. Using the resulting formula for the cross section in parallel kinematics, all of the $\epsilon$ points for a given $Q^{2}$ were then fit to a straight line using linear least-squared fitting. The slope of the line yields the value of $\sigma_{\mathrm{L}}$ while the intercept is the resulting transverse cross section $\sigma_{\mathrm{T}}$.

The separated longitudinal cross section $\sigma_{\mathrm{L}}$ is shown in the top part of Figure (3) while the transverse cross section $\sigma_{\mathrm{T}}$ is shown in the bottom part of the figure, both as functions of $Q^{2}$. Both cross sections show a decrease with increasing $Q^{2}$. While it is expected that the longitudinal cross section increases (with respect to the transverse cross section) in the limit of very high momentum transfers, for the kinematics in E93018, the longitudinal cross section is a decreasing function of momentum transfer. The transverse cross section is expected to be small at very high momentum transfers; this behavior is seen even at the E93-018 $\mathrm{Q}^{2}$ range. The transverse cross section is expected to approach the photoproduction point as $\mathrm{Q}^{2}$ goes to zero ${ }^{17}$. (The ratio of longitudinal to transverse cross sections is expected to be very large at very high momentum transfers.) In the figures, several calculations are shown for comparison with the data. The thin curves correspond to the calculations by the Saclay-Lyon (SL) group ${ }^{18}$ using an isobaric model for the transition form factors. The medium thickness curves correspond to calculations by Williams, $\mathrm{Ji}$, and Cotanch (WJC) ${ }^{19}$, also using an isobaric model, while the thicker weight curves correspond to a Regge model calculation of Vanderhaeghen, Guidal, and Laget (VGL) ${ }^{20}$.

As can be seen, the SL model shows a flat $\sigma_{\mathrm{L}}$ vs $\mathrm{Q}^{2}$ dependence (after the initial rise from $Q^{2}=0$ predicted by all models), in contrast with the behavior exhibited by the data which falls with increasing $Q^{2}$. The WJC calculation shows a pronounced rise of the longitudinal cross sections initially, and then a gradual decrease with increasing $\mathrm{Q}^{2}$, in good agreement with the data. The 

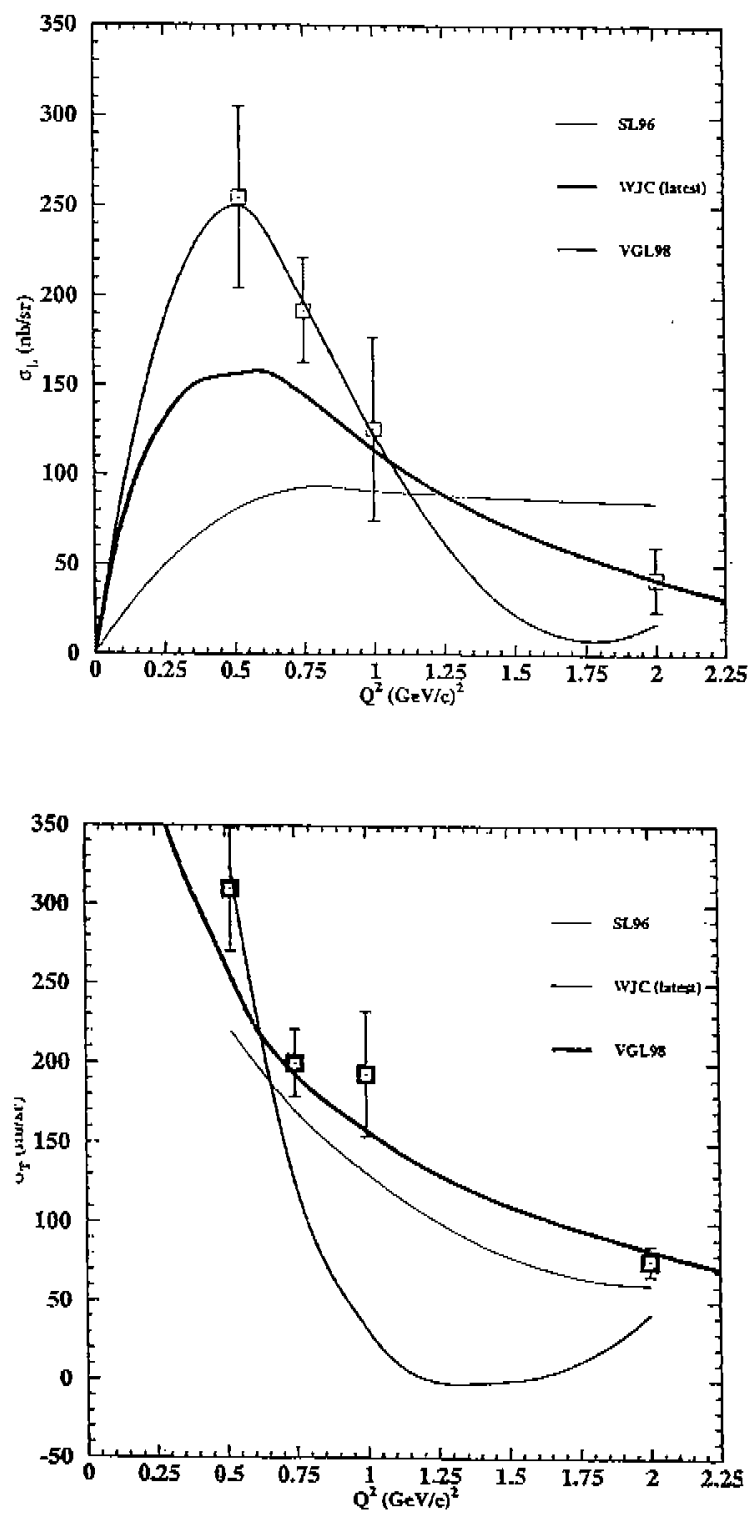

Figure 3. The longitudinal cross section for kaon electroproduction is shown in the top part of the figure while the transverse cross section is shown in the lower part. The thin curves correspond to calculations of the Saclay-Lyon group, the medium thickness curves correspond to the calculations by Williams, $\mathrm{Ji}$, and Cotanch, and the thicker lines show the calculations of Vanderhaegen, Guidal, and Laget as discussed in the text. The figure is taken from the dissertation of G. Niculescu. 
VGL model shows a similar squared four-momentum transfer dependence as the WJC model for the longitudinal cross section, although the maximum of the cross section is not as pronounced in their model calculation.

In the case of the transverse cross section, the SL and the VGL model calculations correctly reproduce the $\mathrm{Q}^{2}$ behavior of the data (although the SL curve is about $10-15 \%$ lower than the data but still consistent with it within the experimental uncertainties of the measurement), whereas the WJC model does not provide as accurate a description of the data as compared to the SL or VGL calculations.

The main differences between the two isobaric model calculations (SL 18 and $\mathrm{WJC}{ }^{19}$ ) is that the former includes resonances up to spin $5 / 2$ whereas the latter includes only up to spin $1 / 2$ hadronic resonances. This difference would show up in a more striking manner in the transverse cross section since it is expected that higher mass and higher spin resonances couple more strongly to the transverse virtual photons. In the longitudinal channel, the cross section is expected to be dominated by the t-channel kaon exchange, and not as sensitive (by comparison with the transverse channel) to $s$ and u channel exchanges. Then it is not unexpected that the WJC model can provide a good description of the trend of the $\sigma_{\mathrm{L}}$ data. The VGL Regge model calculation includes all resonances lying on the appropriate hadron trajectory ${ }^{20}$. This particular model provides a rather good description of the $\mathrm{Q}^{2}$ dependence of both $\sigma_{\mathrm{L}}$ and $\sigma_{\mathrm{T}}$, showing the utility of the Regge description of kaon electroproduction processes. We have also studied the t-dependence in order to extract information about the kaon electromagnetic form factor. These results will be published at a later date ${ }^{17} 21$. It is also intriguing to speculate that the inclusion of even higher spin/mass resonances, or even hidden resonances (which would decay strongly into a kaon and lower mass hyperon) would provide an even better description of the $Q^{2}$ dependences of these separated cross sections ${ }^{17}$. Further experimental results of strangeness production using electrons should shed light on this subject.

\section{Acknowledgments}

This work is supported by an award from the Department of Energy (DEFG05-91ER40620).

\section{References}

1. Gauge Field Theories by M. Guidry John Wiley and Sons, N.Y. (1991).

2. R. Dashen, Phys. Rev 183, 1245 (1969). 
3. G. Knochlein, D. Drechsel, L. Tiator, Z. Phys. A 352, 327 (1995).

4. C. Armstrong, Ph.D. Dissertation, The College of William and Mary, unpublished (1998).

5. Z. Li and F. Close Phys. Rev. D 22; 2157 (1980).

6. M. Warns et.al., Z. Phys. C 45, 627 (1990); Phys. Rev. D' 42, 2215 (1990).

7. S. Capstick and B. Keister, Phys. Rev. D 51, 3598 (1995).

8. R. Stanley and H. Weber, Phys. Rev. C 52, 435 (1995).

9. S.R. Cotanch, R.A. Williams, and C.-R. Ji, Phy. Scripta. 48, 217 (1993).

10. W. Greiner and A. Schafer, Quantum Chromodynamics, Springer-Verlag (1994).

11. C. Bennhold, Nucl. Phys. A 547, 79c (1992); Z. Li, Phys. Rev. C 52, 1648 (1995).

12. C. Bennhold and L.E. Wright, Phys. Rev. C 36, 438 (1987); S.R. Cotanch and S.S. Hsio, Nucl. Phys. A 450, 419c (1986).

13. R.A. Schumacher, Nucl. Phys. A 585, 63c (1995).

14. X.-D. Li et. al., Phys. Rev. Lett. 83, 3776 (1999).

15. R.A. Williams, C.R. Ji, and S.R. Cotanch, Phys. Rev. C 46, 1617 (1992).

16. R.C.E. Devenish and D.H. Lyth, Phys. Rev. D 5, 47 (1972).

17. G. Niculescu, Ph.D. Dissertation, Hampton University, unpublished (1998).

18. J.C. David et. al., Phys. Rev. C 53, 2613 (1996).

19. R.A. Williams, private communications (1998).

20. M. Guidal, J.M. Laget, and M. Vanderhaeghen, Phys. Lett. B 400, 6 (1997);Nucl. Phys. A 627, 645 (1997); M. Vanderhaeghen, M. Guidal, and J.M. Laget,Phys. Rev. C 57, 1454 (1998) .

21. J. Cha, Ph.D. Dissertation, Hampton University, unpublished (2000). 